\title{
Airport Taxi Scheduling Strategy Based on Particle Swarm Optimization Algorithm
}

\author{
Zang Jingnan \\ Institute for Advanced Studies in Humanities and Social \\ Sciences \\ Beijing University of Aeronautics and Astronautics \\ Beijing, China \\ jingnan_zang@sina.cn
}

\author{
Liu Qing \\ College of General Aviation \\ Civil Aviation university of China \\ Tianjin, China \\ lq_hf@sina.cn
}

\begin{abstract}
This paper constructs a new $J_{m} / r_{j} / C$ function of airport taxi-scheduling, and the improved IPSO and CPSO algorithms were introduced to solve these problems. Simulation results showed that the new scheme reduced total time by 25.3 minutes in peak hour, with average 1.4 minutes taxi time reduced per aircraft compared to FCFS strategy. The CPSO algorithm has advantages over the other two algorithms for its acceptable computer time and precise outcomes, which can be used in solving such NP complexity problems.
\end{abstract}

Keywords-Aircraft taxiing;Airport Surface Control;Taxiway Scheduling;Particle Swarm Optimization Algorithm

\section{INTRODUCTION}

Nowadays, hub airport peak hours scheduling problem has become a focused research problem. Researches mainly concentrated on the aircraft taxi-scheduling problems in large hub airport ${ }^{[1-7]}$. J.W. Smeltink and other people came up with the static scheduling mixed integerprogramming model of airport taxi scheduling; the model used the accurate local optimization algorithm which is based on rolling time domain. According to combining with the real evidence of the Amsterdam airport, it shows that this scheduling method solve the problem of optimized scheduling order of different conflict during the taxi schedule, it can improve the efficiency of the operation of the whole airport ${ }^{[1]}$. Ioannis Anagnostakis designed the two-phase planning algorithm of scene taxi schedule problem ${ }^{[2]}$. Balakrishnan and Sivakumar came up with the problem of appointing the best time for each aircraft to depart from the time for arriving on one location during the flight ${ }^{[3]}$. Some domestic scholars begin to pay attention to aircraft scheduling problem. You Jie established the multi Agent model which is decided by the shortest time of taxiing, he fused the optimal path and the thought of contract net protocol, and then came up with the taxi path optimization algorithm which was based on multiple agents ${ }^{[4]}$. Liu Changyou researched the aircraft taxiing security problems; he used the typical conflict restriction rules and safety interval as the constraint, and set up the system conflict-free taxiing path optimization model ${ }^{[5]}$. Job shop scheduling problem provides a way to solve NP complexity problem, many scholars applied typical scheduling to other fields, and received good scheduling efficiency. The typical scheduling includes bus intelligent dispatching ${ }^{[6]}$ and the reservoir optimal operation ${ }^{[7]}$, etc., in these fields; most specific scheduling models are abstracted into the Flow Shop problem, which is easier to solve the problems than shop scheduling. Taxi scheduling is a complex optimization problem; the scheduling process is similar to Job shop problem with many artifacts and variable machines, which is aimed at minimizing the total operation time. But since the number of flights is great and the taxiing way system is enormous in big airport, the modeling of aircrafts scheduling is more complicated. Particle swarm optimization and its improved form have a good time efficiency and convergence of space ${ }^{[8-10]}$; it can be used as a method to solve the new scheduling model.

\section{PROBLEM DESCRIPTION}

When a large number of flights taxi at the same time on the taxi-lane, three taxiing conflicts usually emerge (Fig. 1): (1) Deadlock conflict; (2) Exceeding conflict; (3) Crossconflict.

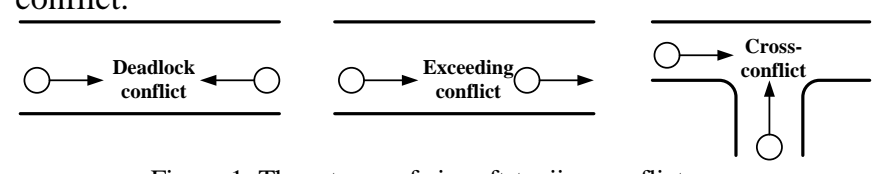

Figure 1. Three types of aircraft-taxiing conflicts

\section{MODELING OF TAXI SCHEDULING}

\section{A. Definition of variable}

Aircraft set is regarded as a set of work piece, taxiway edge set is regarded as a machine set. The taxi scheduling variables definitions are as follows:

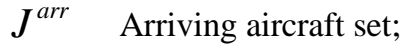
$J^{\text {dep }} \quad$ Departure aircraft set;
$A T A_{i} \quad$ Actual landing time of the flight;
$P B T_{i}$ Time of taxiing into the gate;
$O B T_{i}$ Time of withdrawal of the round file;
$T T D_{i}$ Target time of departure;
$\overline{v_{p}} \quad$ The average velocity of taxiing aircraft;
$d_{i j}^{\text {sep }} \quad$ Minimum taxiing spacing of two aircraft; 
$T_{i j}^{\text {sep }}$ Minimum interval of different type of aircraft;

$d_{i j}^{k}$ Distance on the edge $k$ of aircraft $i$ and $j$;

$$
R_{i}=\left\{r_{1}^{i}, r_{2}^{i}, \cdots, r_{l_{i}}^{i}\right\} \text { Scheduling path set of aircraft } i
$$

$r_{j}^{i}$ represents the $j$ edge of its taxiing path;

$t_{i}^{r_{j}}$ Represents the taxiing time on the $k$ edge of taxiing path $R$ of aircraft $i$;

$y_{i}^{k}=1$, Aircraft $\mathrm{i}$ arrived at taxiing path $\mathrm{k}$ before aircraft $\mathrm{j}$

0,Otherwise

$z_{i j}^{k}=1$, Aircraft $\mathrm{i}$ used taxiing path $\mathrm{k}$ before aircraft $\mathrm{j}$ 0,Otherwise

Among them, $J=J^{a r r}+J^{d e p}, \quad R_{i} \subseteq E$.

\section{B. Mathematical model}

Objectives function-minimize the time of all aircraft taxiing:

$$
\min _{t} Z_{J}=\sum_{i \in J^{d e p}}\left(T T D_{i}-t_{i}^{r_{1}}\right)+\sum_{i \in J^{a r r}}\left(t_{i}^{r_{k_{i}}}-t_{i}^{r_{i}}\right)
$$

Constraint_-include the taxiing order, capacity, safety interval, and arriving/departure time:

Taxiing order constraint:

$$
y_{i j}^{k}+y_{j i}^{k}=1 \forall i, j \in J, k \in R_{i} \cap R_{j}, i \neq j
$$

Constraint (2) describes the order of two aircraft $i$ and $j$

Capacity constraint:

$$
z_{i j}^{k}+z_{j i}^{k}=1 \quad \forall i, j \in J, i \neq j
$$

Constraint (3) describes only one aircraft allowed on the edge of taxiway at the same time.

Conflict and interval constraints:

$$
\begin{aligned}
& y_{i j}^{k}\left(d_{i j}^{k}-d_{i j}^{s e p}\right) \geq 0 \forall i, j \in J, k \in R_{i} \cap R_{j}, i \neq j(4) \\
& t_{j}^{r_{k_{j}}}-t_{i}^{r_{k_{i}}}-T_{i j}^{s e p} \geq-\left(1-z_{i j}^{k}\right) M \quad \forall i, j \in J^{d e p}, i \neq j(5)
\end{aligned}
$$

Constraint (4) and (5) describes the minimum safety distance and interval time in same taxiing lane.

Time constraints:

$$
\begin{array}{cl}
t_{i}^{r_{k_{i}}} \geq T T D_{i} & \forall i \in J^{d e p}, k \in R_{i} \\
t_{i}^{r_{k_{1}}} \geq O B T_{i} & \forall i \in J^{d e p}, k \in R_{i} \\
t_{i}^{r_{k i}} \leq A T A_{i} & \forall i \in J^{\text {arr }}, k \in R_{i} \\
t_{i}^{r_{k 1}} \leq P B T_{i} & \forall i \in J^{\text {arr }}, k \in R_{i}
\end{array}
$$

Constraint (6) - (9) describes an aircraft scheduling must satisfy the arranged takeoff and landing time.

Other constraints:

$$
\begin{gathered}
y_{i j}^{k}, z_{i j}^{k} \in\{0,1\} \quad \forall i, j \in J, i \neq j, k \in R_{i} \cap R_{j} \\
t_{i}^{r_{k_{i}}} \in R^{+}, \forall i \in J, k \in R_{i}
\end{gathered}
$$

Constraints (10) - (11) describes decision variables.

\section{PARTICLE SWARM OPTIMIZATION ALGORITHM}

\section{A. The improved particle swarm algorithm}

Basic Particle swarm optimization (PSO) is a Swarm intelligence optimization algorithm, which Kennedy and Eberhart came up with it in 1995. The standard formula of the particle is ${ }^{[16]}$

$$
\begin{gathered}
v_{i d}^{t+1}=\omega \times v_{i d}^{t}+c_{1} \times r_{1} \times\left(\text { pbest }_{i d}^{t}-x_{i d}^{t}\right)+c_{2} \times r_{2} \times\left(\text { gbest }_{i d}^{t}-x_{i d}^{t}\right) \\
x_{i}^{t+1}=x_{i}^{t}+v_{i}^{t}
\end{gathered}
$$

(12) And (13) calculate the speed and position of the particle at dimension and at time of $t+1 . v_{i}$ is the speed of the particle, $\omega$ is called inertia weight, $x_{i}$ refers to the current position of the particle, $c_{1}$ and $c_{2}$ are learning factors, $r_{1}$ and $r_{2}$ are random numbers between $(0,1)$.

\section{Plan 1: Increase the improved particle swarm} optimization (IPSO)

The compression factor helps to ensure PSO algorithm converge, the speed of particle updates equation as:

$$
\begin{gathered}
v_{i d}^{t+1}=k\left(\omega \times v_{i d}^{t}+c_{1} \times r_{1} \times\left(\text { pbest }_{i d}^{t}-x_{i d}^{t}\right)+c_{2} \times r_{2} \times\left(\text { gbest }_{i d}^{t}-x_{i d}^{t}\right)\right) \\
\text { In formula (14) }), \quad k=2 /\left|2-l-\sqrt{l^{2}-4 l}\right|,
\end{gathered}
$$
$l=c_{1}+c_{2}, l \geq 4$, and as compression factor, $k$ is used to control the weight of speed. pbest is optimal position that the particle itself has experienced, gbest is the optimal position that particle swarm has experienced. Compression factor can be used to control system converge. In order to obtain high quality solutions, it can search different regions effectively.

\section{Plan 2: Cooperative particle swarm optimization (CPSO)}

Classic particle swarm algorithm is easy to have a "premature" phenomenon; it limits the searching scope of particles. Collaborative search algorithm divides all particles into several sub populations, and uses multiple independent subgroup for collaborative optimization, it is divided into two layers structure, the underlying make multiple particle swarm search the space of solution independently in order to expand the search scope; the upper particle swarm chase the current global optimal solution.

\section{B. Improved PSO algorithm to solve the scheduling problem}

1) Encoding mechanism. Since scheduling is based on the operation, it is similar to the coding of a shop scheduling that is based on process operation ${ }^{[16]}$; the order of the particle represents the scheduling sequence. The $d=n \times m$ represents position vector of particles, a particle can be expressed as dimensional $d$, and each aircraft will appear $m$ times. Taking an example of $3 / 5 / Z_{J}$ problem, three aircraft taxiing on the five taxiing side, then the 
position

$$
X=[1,2,3,4,5,2,3,1,5,4,4,1,5,3,2]
$$

explain $J_{1}-J_{2}-J_{3}$ is the passing order of path $R_{1}$ and $J_{2}-J_{3}-J_{1}$ is the passing order of path 2 according to the aircraft taxiing order.

Table I AIRCRAFT TAXIING ORDER

\begin{tabular}{cccccc}
\hline \multirow{2}{*}{ Aircraft } & \multicolumn{5}{c}{ Taxiing order } \\
\cline { 2 - 6 } & Path 1 & Path 2 & Path 3 & Path 4 & Path 5 \\
\hline$J_{1}$ & $R_{1}$ & $R_{2}$ & $R_{3}$ & $R_{4}$ & $R_{5}$ \\
$J_{2}$ & $R_{2}$ & $R_{3}$ & $R_{1}$ & $R_{5}$ & $R_{4}$ \\
$J_{3}$ & $R_{4}$ & $R_{1}$ & $R_{5}$ & $R_{3}$ & $R_{2}$ \\
\hline
\end{tabular}

2) Fitness function. Fitness function is used to evaluate the performance of the particle, the goal of the function is to minimize the scheduling time. Safety interval factors must be considered as constraint (5) shows. The algorithm treats the objective function

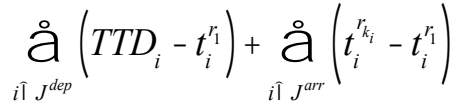
$-M \times \operatorname{Min}\left[\left(t_{j}^{r_{k_{j}}}-t_{i}^{r_{k_{i}}}-T_{i j}^{s e p}\right), 0\right]$ as the fitness function, among them $-M \times \operatorname{Min}\left[\left(t_{j}^{r_{k_{j}}}-t_{i}^{r_{k_{i}}}-T_{i j}^{\text {sep }}\right), 0\right]$ is in the penalty term of the fitness function as constraint, once the minimum safety interval is less than the specified value, then added the infinite positive item into the fitness function of the particle can avoid the next iteration.

3) The evolutionary strategy. Adopt the strategy of compression factor $k$, it makes the algorithm can converge faster. The update of particles is based on formula (14). The steps of using the algorithm of plan 1 to solve aircraft scheduling model are as following:

Step 1: Determine the takeoff and landing sequence according to the time information T_flight table, using the shortest path algorithm to initialize the scheduling path, then calculate the ideal taxiing time of each aircraft in their respective path set $R_{i}$.

Step 2: Initialize the parameter, and calculate the initial target.

Step 3: Compare the fitness function value $Z_{\text {temp }}$ that calculated in step 2 with the optimal value of the current fitness, if the latter were better, then the new fitness function value would take place of the previous one. Use the corresponding new particles to take place of the original particles to get the individual optimal solution $X_{p_{i}}$ of each particle;

Step 4: Use the formula (12) and (13) to move particles to produce a new particle swarm, and then repeat steps 2 to 5, until iterations are completed.

Step 5: Calculate the scheduling time, and draw the scheduling Gantt chart, then get the scheduling plan.

The steps of plan 2 algorithm to solve aircraft scheduling model are as following:

Step 1: The same as plan 1;
Step 2: Set up two layers of particle parameters, one global optimal particle population and 5 sub population, and calculate the initial target. ,

Step 3: Find optimal fitness function value $Z_{\text {temp }}$ from the five sub populations and then compare them with the optimal value of the current global optimal fitness, if $Z_{\text {temp }}$ were better, then use the new fitness function value instead of the previous one. Use the corresponding new particles instead of the original one; and get the individual optimal solution $X_{p_{i}}$ of each particle;

Step 4: 5 sub population are reproduced on the basis of the optimal population, use the corresponding new particles to take place of the original particles, and repeat step 2 to 3 until iterations are completed.

Step 5: Calculate the scheduling time, and draw the schedule Gantt chart, then get the scheduling scheme.

\section{SIMULATE EXPERIMENT}

\section{A. Basic data}

Choose one-day flight plan of a large domestic hub airport as the research object, that airport runway has two parallel taxiway and more than 80 slots. Consider the influence of drafting, the aircraft should keep certain safety interval on the taxiway, different types of the aircraft need to keep the safety interval which is not less than 43 seconds interval so that it can avoid conflict.

\section{B. Solution of the model and the result analysis}

In order to increase the high accuracy and stability, the size of particle population is set to $80^{[18]}$ and take $c_{1}=c_{2}=2, k=0.729,300$ largest iterative and $\omega$ decreased from 0.9 to 0.4 . Use the most short-circuit algorithm to obtained the taxiing order of 18 aircraft in the taxiway, which is shown in table 2.

The simulate results shows that optimal time respectively reduces 178 second, 1607 second and 1519 second compared with the strategy of actual time. First come first service (FCFS) is used generally, the route of aircraft are relatively fixed. IPSO algorithm reduced overall interval flight time, and made fully use of the resources and capacity of the airport, also improve the efficiency of scheduling greatly.

The results of three kinds of particle swarm optimization (PSO) algorithm show that PSO and IPSO algorithm can obtain satisfactory solution in relatively short period of time and CPSO can get the optimal solution for short scheduling plan; PSO and IPSO method are not stable when the scale exceed more than 10 aircraft, the value calculated by IPSO is slightly better than the one that from PSO. CPSO was more time efficiency and accuracy when the scale is more than 18 aircraft.

Fig. 2 shows it is easier for CPSO to get the global optimal solution than IPSO and PSO algorithm. According to the simulation of aircraft scheduling in the peak hour, the optimal solution of basic PSO algorithm is 1397 seconds, the solution of IPSO algorithm is 1360 seconds, and both two algorithms are easy to fall into local optimum. Only 
CSPO algorithm has the strong ability of particle control, the solution of 1186 seconds is the global optimal solution, and the solution has the stable quality.
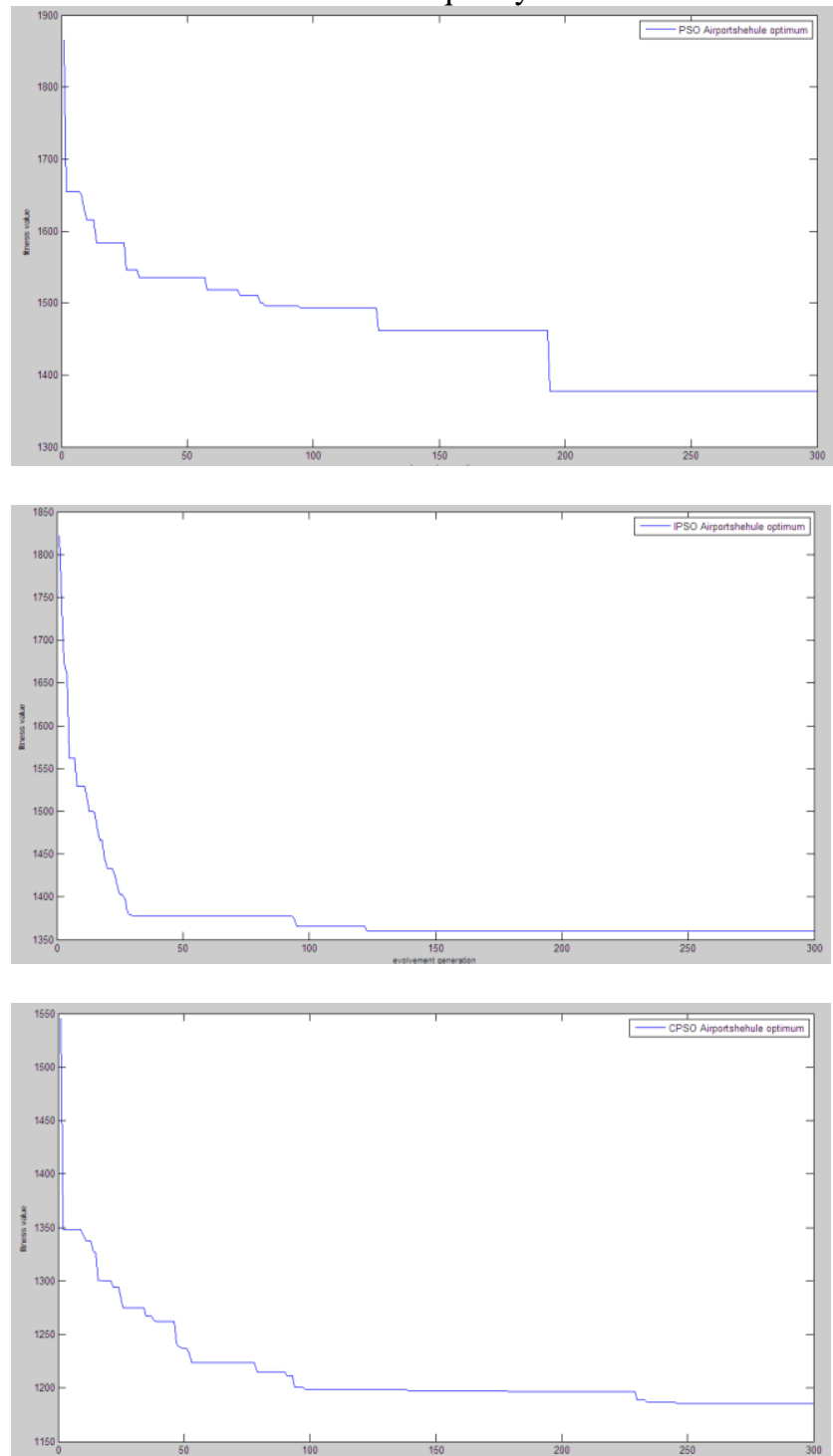

Figure 2. Comparisons of PSO, IPSO and CPSO

\section{CONCLUSIONS}

In this paper the problem of aircraft scheduling in the peak hour is launched. According to the results, the time of aircraft scheduling during the peak hour in a hub airport can be reduced by 25.3 minutes, each aircraft can save 1.4 minutes, which take advantage of FCFS plan and has significance of improving capacity of airport. From solutions of the model, cooperative particle swarm reveals time efficiency and precision in solving the problems.

\section{ACKNOWLEDGMENT}

This research was financially supported by the Basic scientific research projects of the Central University of China (ZXH2012L004).

\section{REFERENCES}

[1] J.W. Smeltink, M.J. Soomer, P.R.DeWaal and Van Der Mei, R.D. "An Optimisation Model for Airport Taxi Scheduling"[C]// Thirtieth Conference on the Mathematics of Operations Research, Lunteren, the Netherlands, 2005, pp.1-25.

[2] I. Anagnostakis, and J-P. Clarke, Runway Operations Planning: A Two-Stage Solution Methodology, Proc. of the 36th Hawai International Conference on System Sciences (HICSS'03). 2003.

[3] Balakrishnan, H., and Jung, Y., "A Framework for Coordinated Surface Operations Planning at Dallas-Fort Worth International Airport", AIAA Guidance, Navigation, and Control Conferen- ce Hilton Head, SC, August 20-23, 2007.

[4] You Jie, Han Songchen. Taxi route optimization algorithm of airport surface based on multi-agent $[\mathrm{J}]$. Journal of Traffic and Transportation Engineering, 2009, vol. 2. pp.109-113.

[5] Liu Changyou, Chong Xiaodong. The optimization of aircraft taxiing based on GA arithmetic $[\mathrm{J}]$. Journal of Transportation Information and Safety, 2009, 3(27):6-10.

[6] Natalia VS,Yuri NS,Frank W.Complexity of mixed shop scheduling problems European Journal of Operational Research,2000, vol. 120. pp.343-351.

[7] Hoitomt DJ,Luh PB,and Pattipafi KR.Job shop scheduling. In Proc.1 “Int.Conf.Automat.Techn01.,Talpei,Taiwan,1990, pp.565574.

[8] Eberhart R C, Kennedy J. A new optimizer using particles swarm theory $[\mathrm{C}]$. Proceedings 6th International Symposium on Micro Machine and Human Science.Nagoya,Japan,1995, pp.3943.

[9] Natalia VS,Yuri NS,Frank W.Complexity of mixed shop scheduling problems European Journal of Operational Research,2000,120:343-351.

[10] Hoitomt DJ,Luh PB,and Pattipafi KR.Job shop scheduling. In Proc.1 Int.Conf.Automat.Techn01.,Talpei,Taiwan,1990:565-574.

Table II . OPTIMIZATION OF COMPARISION OF FLIGHTS SCHEDULING PLAN (UNIT:S)

\begin{tabular}{llllllll}
\hline \multirow{2}{*}{ Taxing schedule } & \multirow{2}{*}{ FCFS } & \multicolumn{2}{c}{ PSO } & \multicolumn{2}{c}{ IPSO } & \multicolumn{2}{c}{ CPSO } \\
\cline { 3 - 8 } & & optimal & time & optimal & time & optimal & time \\
\hline AS06 $(6 \times 37)$ & 906.2 & 728.2 & 5.321155 & 728.2 & 5.320158 & 728.2 & 28.850980 \\
AS12 $(12 \times 37)$ & 2585.4 & 1055 & 9.901543 & 1043 & 9.862927 & 978.1 & 54.501568 \\
AS18 $(18 \times 37)$ & 2705.4 & 1397 & 14.930866 & 1360 & 14.539424 & 1186 & 81.348122 \\
\hline
\end{tabular}

